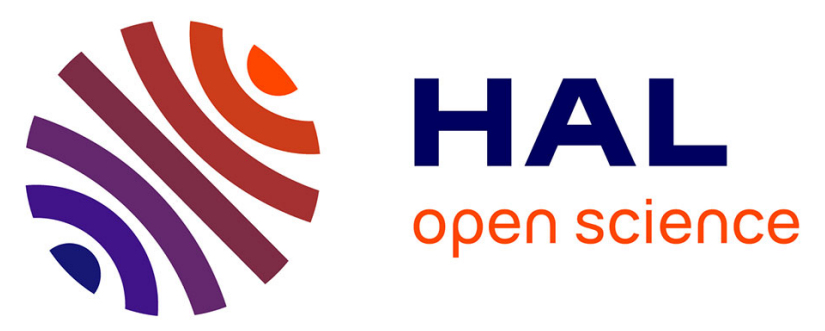

\title{
Validation of hip joint center localization methods during gait analysis using 3D EOS imaging in typically developing and cerebral palsy children
}

Ayman Assi, Christophe Sauret, Abir Massaad, Ziad Bakouny, Hélène Pillet, Wafa Skalli, Ismat Ghanem

\section{To cite this version:}

Ayman Assi, Christophe Sauret, Abir Massaad, Ziad Bakouny, Hélène Pillet, et al.. Validation of hip joint center localization methods during gait analysis using 3D EOS imaging in typically developing and cerebral palsy children. Gait \& Posture, 2016, 48, pp.30-35. 10.1016/j.gaitpost.2016.04.028 . hal-02181648

\section{HAL Id: hal-02181648 \\ https://hal.science/hal-02181648}

Submitted on 12 Jul 2019

HAL is a multi-disciplinary open access archive for the deposit and dissemination of scientific research documents, whether they are published or not. The documents may come from teaching and research institutions in France or abroad, or from public or private research centers.
L'archive ouverte pluridisciplinaire HAL, est destinée au dépôt et à la diffusion de documents scientifiques de niveau recherche, publiés ou non, émanant des établissements d'enseignement et de recherche français ou étrangers, des laboratoires publics ou privés. 


\title{
Validation of hip joint center localization methods during gait analysis using 3D EOS imaging in typically developing and cerebral palsy children
}

\author{
Ayman Assi ${ }^{\mathrm{a}, \mathrm{b}, \mathrm{c}, *}$, Christophe Sauret $^{\mathrm{b}}$, Abir Massaad ${ }^{\mathrm{a}, \mathrm{c}}$, Ziad Bakouny $^{\mathrm{a}}$, Hélène Pillet ${ }^{\mathrm{b}}$, \\ Wafa Skalli ${ }^{\mathrm{b}}$, Ismat Ghanem ${ }^{\mathrm{a}, \mathrm{c}, \mathrm{d}}$
}

a Laboratory of Biomechanics and Medical Imaging, Faculty of Medicine, University of Saint-Joseph, Beirut, Lebanon

${ }^{\mathrm{b}}$ Institut de Biomécanique Humaine Georges Charpak, Arts et Métiers ParisTech, Paris, France

' Gait Laboratory, SESOBEL, Beirut, Lebanon

${ }^{\mathrm{d}}$ Hôtel-Dieu de France Hospital, Beirut, Lebanon

Keywords:

Hip joint center

Cerebral palsy

EOS biplanar X-rays

Gait analysis

Children

Localizationofthehipjointcenter(HJC)isessentialincomputationofgaitdata.EOSlowdosebiplanar X-rayshavebeenshowntobeagoodreferenceinevaluating various methods of $\mathrm{HJClocalization} \mathrm{in} \mathrm{adults.} \mathrm{The} \mathrm{aim} \mathrm{is} \mathrm{to} \mathrm{evaluate} \mathrm{predictive} \mathrm{and} \mathrm{functional} \mathrm{techniques} \mathrm{for} \mathrm{HJClocalization} \mathrm{in} \mathrm{typically} \mathrm{developing(TD)} \mathrm{and}$ cerebral palsy $(\mathrm{CP})$ children, usingEOSas an image based reference. ElevenTDand 17CPchildrenunderwent3Dgaitanalysis.SixHJClocalization methodswere evaluatedineachgroup bilaterally:3 predictive(PluginGait,BellandHarrington)and 3 functionalmethodsbasedonthestararc technique(symmetricalcenter of rotation estimate, center transformation technique and geometrical sphere fitting). All children then underwentEOSlow dose biplanar radiographs. Pelvis, lower limbs and their corresponding external markers were reconstructed in $3 \mathrm{D}$. The center of the femoral head was considered as the reference ( $\mathrm{HJC} \mathrm{E}_{\mathrm{EOS}}$ ). Euclideandistances between $\mathrm{HJCsestimatedbyeach} \mathrm{of} \mathrm{the} 6$ methodsand the $\mathrm{HJC}_{\mathrm{EO}}$ werecalculated; distanceswereshowntobelowerinpredictivecompared to functional methods $(p<0.0001)$. Contrarily to findings in adults, functional methods were shown to be less accurate than predictive methods in TD and CP children,whichcouldbemainlyduetotheshorter thighsegmentinchildren.Harringtonmethodwasshowntobethemostaccurateinthepredictionof $\mathrm{HJC}$ (mean error $\approx 18 \mathrm{~mm}, \mathrm{SD}=9 \mathrm{~mm}$ ) and quasi-equivalent to the Bell method. The bias for each method was quantified, allowing its correction for an improved $\mathrm{HJC}$ estimation.

\section{Introduction}

Motion analysis has become an important asset during decision making for the treatment of musculoskeletal disorders, such as cerebral palsy [1,2]. Gait analysis is based on biomechanical models that use external skin markers in order to calculate joint kinematics and kinetics $[3,4]$. These external markers are used to associate a local coordinate system to each skeletal segment. The hip joint center $(\mathrm{HJC})$ is an essential landmark for the creation of the local coordinate system of the thigh segment [5]. While this anatomical landmark is not directly accessible externally, a virtual marker is associated to this point. Two possible approaches exist in

\footnotetext{
* Corresponding author at: Faculty of Medicine, University of Saint-Joseph, Damascus Street, Campus of Innovation and Sport, Beirut, Lebanon. Tel: +9611421676; fax: +9611421681.

E-mail addresses: ayman.assi@gmail.com, ayman.assi@usj.edu.lb (A. Assi).
}

the localization of the HJC: either predictive techniques based on regression equations using anthropometric measurements [6-8] or functional calibration methods based on the movement of the thigh relative to the pelvis, where the center of rotation is determined [9-13].

The validation of these hip joint center localization techniques has been mainly based on cadaveric experiments or simulations $[11,14]$. Medical imaging techniques could be used in order to validate the HJC localization methods in vivo. Fluoroscopy has been previously used in some of the validation studies [9,15]. However, this method is known to deliver a considerable dose of radiation to the patient. Other studies have used three-dimensional (3D) ultrasound and have shown it to be effective $[16,17]$. However, this method cannot allow simultaneous acquisitions of both HJC and external markers. Magnetic Resonance Imaging could be an alternative [8], but is costly and time consuming. 
More recently, the EOS ${ }^{\circledR}$ system, which is a low dose biplanar radiographic system [18], has been shown to be accurate and reliable in both the detection of the reference HJC and the external markers [19]. The advantage of this technique is that it allows 3D reconstructions of the skeletal segments based on simultaneous biplanar radiographs taken from head to feet while the subject is in a standing position. The $\mathrm{EOS}^{\circledR}$ technique was recently used in order to validate hip joint center localization in healthy adults [20]. However, there are no studies on the validation of the hip joint center localization methods in children using this imaging technique. Thus, the aim was to validate hip joint center localization techniques using the $\operatorname{EOS}^{\circledR}$ system as a 3D image based reference, both in typically developing children and in children with cerebral palsy.

\section{Methods}

\subsection{Population}

After approval from the ethics committee of our institution, seventeen children with cerebral palsy $(6 \mathrm{~F}, 11 \mathrm{M})$ and eleven typically developing children $(6 \mathrm{~F}, 5 \mathrm{M})$ were enrolled in this study. The demographics of both groups were reported in Table 1. All participants and their parents were previously informed of the protocol and signed a written informed consent form prior to their participation in this study, allowing both collection and use of the data reported in this manuscript.

\subsection{Motion capture}

Subjects were equipped with 24 reflective skin markers on their lower limbs, based on the Helen Hayes protocol [7], with additional markers located on the posterior and anterior area of the thigh and the medial femoral condyle. Three-dimensional motion analysis was obtained using seven MX3 cameras (Vicon ${ }^{\circledR}$, Oxford Metrics, UK) and the Workstation ${ }^{\circledR}$ software. First, a static calibration was performed for each subject. The thigh marker was adjusted in order to avoid cross-talk from the knee flexion/extension curve to the knee varus/valgus curve, often observed during gait: a qualitative assessment of the alignment of the knee joint center (obtained by the Plug in Gait model) with the lateral and medial condyles was performed. When the knee joint center was found to be anterior (posterior) to the inter-condylar line, the lateral marker of the thigh was shifted anteriorly (posteriorly, respectively). The static calibration was repeated in order to verify the alignment of the knee joint center with the inter-condylar line. Subsequently, the final trial, with the adjusted thigh marker positioning, was used for the study.

Then, the star arc acquisition was performed for each hip using the method previously developed by Camomilla et al. [21]. Each subject had been accustomed to the protocol prior to the acquisitions and a cane was used for stability when necessary. Hip calibration was performed with a maximal comfortable range of motion for the subject. The range of motion (ROM) performed during the star arc acquisition was collected for each subject.

Three predictive methods were used in order to calculate the hip joint center in each subject bilaterally: Plug in Gait (PiG) [7], Bell [6] and Harrington (HAR) [8]. These methods used anthropometric measures such as pelvic measurements and leg length.
Moreover, three functional methods were used in order to calculate the hip joint center bilaterally based on the star arc acquisition: symmetrical center of rotation estimate (SCoRE) [11], center transformation technique (CTT) [12] and geometrical sphere fitting (GSF) [13]. The coordinates of the HJCs obtained from the 3 predictive and 3 functional methods were expressed in the local coordinate system of the pelvis based on the external reflective markers [7].

\subsection{Imaging technique}

All subjects underwent an $\operatorname{EOS}^{\circledR}$ (EOS Imaging, Paris, France) biplanar X-ray [18] examination of their lower limbs (pelvis to feet) following the motion capture acquisitions, with the external reflective markers still in place. Three-dimensional positions of the markers were determined by manual positioning and fitting on frontal and lateral radiographs of a $14 \mathrm{~mm}$ marker model. Subjectspecific 3D reconstructions of the lower limbs were obtained by a method based on simultaneous adjustment of parametric models on frontal and lateral radiographs $[22,23]$. An example of $\operatorname{EOS}^{\circledR}$ biplanar X-rays with 3D reconstruction of the lower limbs and of the reflective markers is shown in Fig. 1. Three-dimensional reconstructions of the femurs were then processed using Stereos research software (Arts et Métiers ParisTech, Paris, France) allowing the acquisition of the femur mesh and embedded regions, including the femoral head. Then, a sphere was fitted to the femoral head region and its center was considered as the reference hip joint center $\left(\mathrm{HJC}_{\mathrm{EOS}}\right)$. $\mathrm{HJC}_{\mathrm{EOS}}$ of the right and left lower limbs were expressed in the pelvic coordinate system based on the external markers of the pelvis seen on the radiographs. This method was previously validated [19] and was shown to be reliable for the detection of external reflective markers (within $0.15 \mathrm{~mm}$ ) and accurate for the detection of the hip joint center (mean errors: $2.9 \mathrm{~mm}$, SD: $1.3 \mathrm{~mm}$ ).

Subsequently, all hip joint centers obtained from predictive and functional methods, as well as the reference $\mathrm{HJC}_{\mathrm{EOS}}$, were all expressed in the same coordinate system.

\subsection{Statistics}

The error on each hip joint center localization technique was evaluated by calculating the Euclidean distance between the evaluated $\mathrm{HJC}$ and the reference $\mathrm{HJC}_{\mathrm{EOS}}$. The proportion of hips falling within the threshold of $30 \mathrm{~mm}[20,24]$ was counted for each localization method. The deviation from the reference in each direction was also calculated: antero-posteriorly, medio-laterally and vertically.

The distribution of all variables was tested for normality using the Shapiro-Wilk test.

First, between-group comparisons (CP vs. TD) of errors on hip joint centers were performed in order to test if the precision of the method differed between groups. The equality of variances was evaluated using Levene's test. Distances to the $\mathrm{HJC}_{\mathrm{EOS}}$ obtained for $\mathrm{CP}$ and TD groups were compared using Student, Mann-Whitney or Welch tests.

Second, between-method comparisons of errors on hip joint centers were performed in order to determine which method is

Table 1

Demographic table of children with cerebral palsy and typically developing children.

\begin{tabular}{|c|c|c|c|c|c|c|}
\hline Groups & $N$ & Age (years) & Weight (kg) & Height $(\mathrm{cm})$ & BMI $\left(\mathrm{kg} / \mathrm{m}^{2}\right)$ & GMFCS levels \\
\hline $\mathrm{CP}$ & 17 & $11.9 \pm 3.5$ & $41.5 \pm 16.8$ & $145.0 \pm 16.3$ & $19.0 \pm 5.0$ & $\begin{array}{l}\text { I: } N=11 \\
\text { II: } N=5 \\
\text { III: } N=1\end{array}$ \\
\hline TD & 11 & $10.7 \pm 2.3$ & $43.7 \pm 14$ & $143.2 \pm 11.0$ & $20.9 \pm 4.1$ & \\
\hline
\end{tabular}




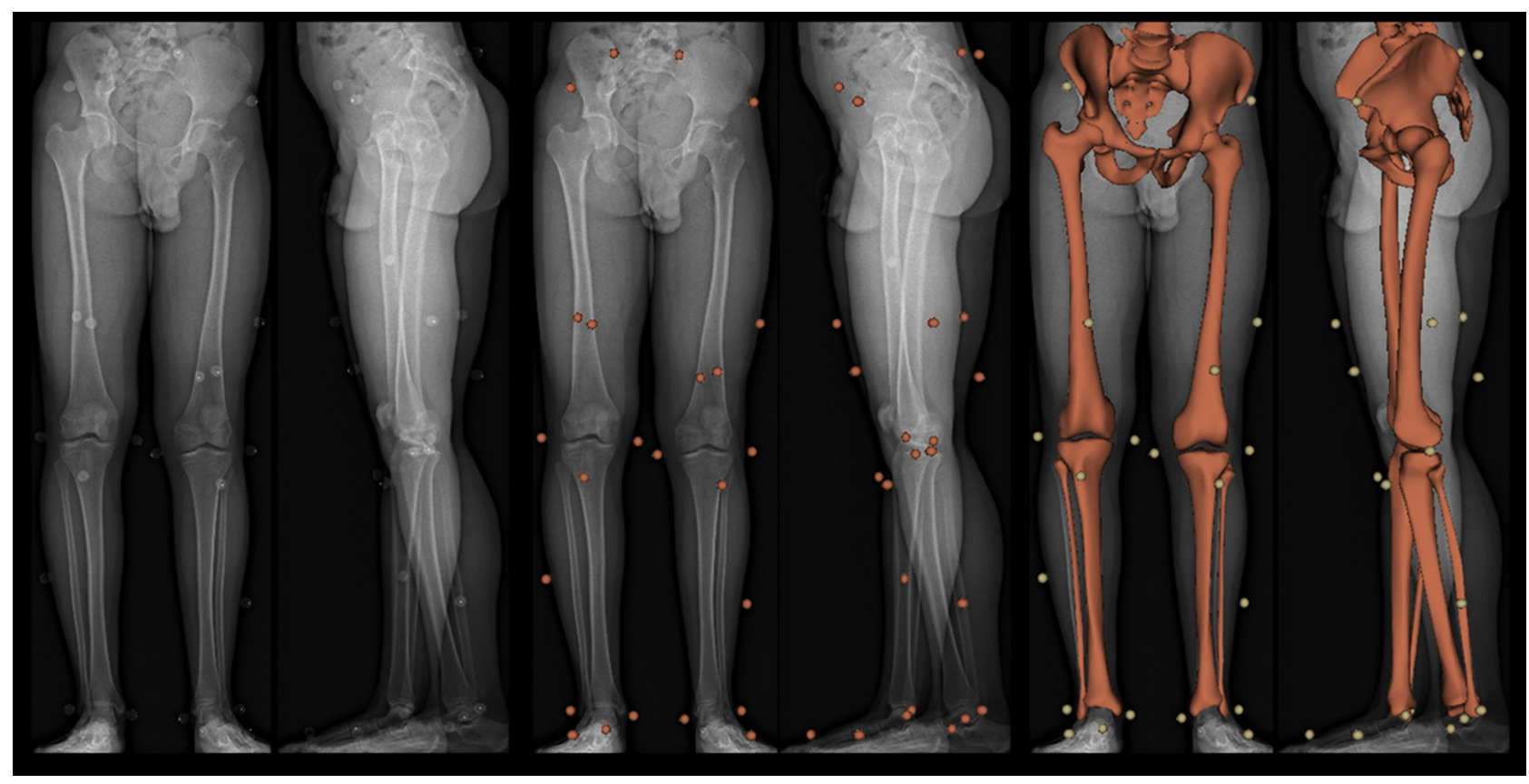

Fig. 1. Frontal and lateral EOS radiographs (left) with 3D reconstruction of the external markers (middle) and of the lower limbs and pelvis (right).

best for $\mathrm{HJC}$ estimation in each group. Data sphericity was evaluated using Mauchly's test. Between-method comparisons ( 3 predictive and 3 functional) of distances to the reference were performed using repeated measures ANOVA with GreenhouseGeisser correction or Friedman's test, with multiple pairwise comparisons using Bonferroni or Nemenyi methods, respectively.

The ROM performed during the star arc acquisition were compared between $\mathrm{CP}$ and TD groups using unpaired $t$-test. A oneway analysis of covariance was performed in order to detect if the ROM was a confounding factor on the errors on hip joint centers calculated by the functional methods.

Level of significance was set at 0.05 . Statistical analysis was performed using Xlstat $^{\circledR}$ (version 2015.5.01.22537; Addinsoft, Paris, France) and SPSS Statistics (version 20.0; IBM Corporation, New York, USA) and data were processed using Matlab ${ }^{\circledR}$ (version R2011a; The Mathworks Inc., Natick, MA, USA).

\section{Results}

In total, both hips of all the enrolled children were processed for the predictive methods: 34 hips of children with cerebral palsy (CP) and 22 hips of typically developing (TD) children. Nine CP and 1 TD children were not able to perform the star arc acquisition and were excluded from the database of functional methods; consequently, 16 hips of children with CP and 20 hips of TD children were evaluated for the functional methods.

The distances between the hip joint centers (HJCs) and the $\mathrm{HJC}_{\mathrm{EOS}}$ reference were grouped in Fig. 2.

Comparison of errors on HJC localization techniques between $C P$ and TD groups showed a significant difference for the Bell $(p<0.001)$ and geometrical sphere fitting $(p=0.031)$ methods. Thus, each group was considered separately when comparing the HJC localization methods.

In the TD group (Fig. 3a), a significant difference was found between predictive and functional methods $(p<0.001)$ with lower error for predictive methods. Comparisons between functional methods did not show a significant difference $(p=0.086)$. However, comparisons between predictive methods showed that the Harrington method had significantly lower errors $(19 \pm 9 \mathrm{~mm})$ compared to the Plug in Gait $(25 \pm 10 \mathrm{~mm}, p=0.048)$ and Bell $(23 \pm 8 \mathrm{~mm}, p=0.03)$ methods.

In the CP group (Fig. 3b), a significant difference was found between the predictive and the functional methods $(p<0.001)$, where the predictive methods showed lower mean errors compared to the functional methods. Comparisons between functional methods did not show a significant difference $(p=0.611)$. However, comparisons between predictive methods showed that the Bell method had significantly lower errors $(16 \pm 8.5 \mathrm{~mm})$ compared to the Plug in Gait method $(21 \pm 10 \mathrm{~mm}$, $p=0.005$ ). There was no significant difference between the Bell and Harrington methods ( $p=0.127)$.

The proportions of estimated hip localization errors that were higher than the threshold of $30 \mathrm{~mm}$ ranged between $21 \%$ and $26 \%$ for the predictive methods and between $94 \%$ and $100 \%$ for the functional methods.

The deviations of $\mathrm{HJCS}$ to $\mathrm{HJC}_{\mathrm{EOS}}$ in each direction of the pelvis coordinate system were reported in Fig. 4. While the medio-lateral deviation did not show a specific trend, it was noted that the PiG method tended to shift the HJC posteriorly to the reference $(-17 \pm 10 \mathrm{~mm})$. The functional methods tended to place the HJC anteriorly (SCoRE: $+30 \pm 25 \mathrm{~mm}$, CTT: $+22 \pm 30 \mathrm{~mm}, \quad$ GSF: $+35 \pm 30 \mathrm{~mm}$ ) and superiorly (SCoRE: $+38 \pm 29 \mathrm{~mm}$, CTT: $+38 \pm 30 \mathrm{~mm}$, GSF: $+44 \pm 18 \mathrm{~mm}$ ) to the reference.

The range of motion (ROM) performed during the star arc acquisition was significantly lower $(p=0.02)$ in the CP group compared to the TD group ( $35 \pm 9^{\circ}$ vs. $42 \pm 8^{\circ}$, respectively). The ANCOVA showed that ROM was not a confounding factor for the errors on $\mathrm{HJC}$ localization techniques obtained by the functional methods (SCoRE: $p=0.41$, CTT: $p=0.26$, GSF: $p=0.47$ ).

\section{Discussion}

Three predictive and 3 functional hip joint center localization techniques, used in gait analysis, were compared to the hip joint center obtained by $3 \mathrm{D}$ EOS $^{\circledR}$ imaging in children with cerebral palsy and typically developing children. All predictive methods were shown to be more accurate than the functional methods. Among the predictive methods, the Harrington method was found 

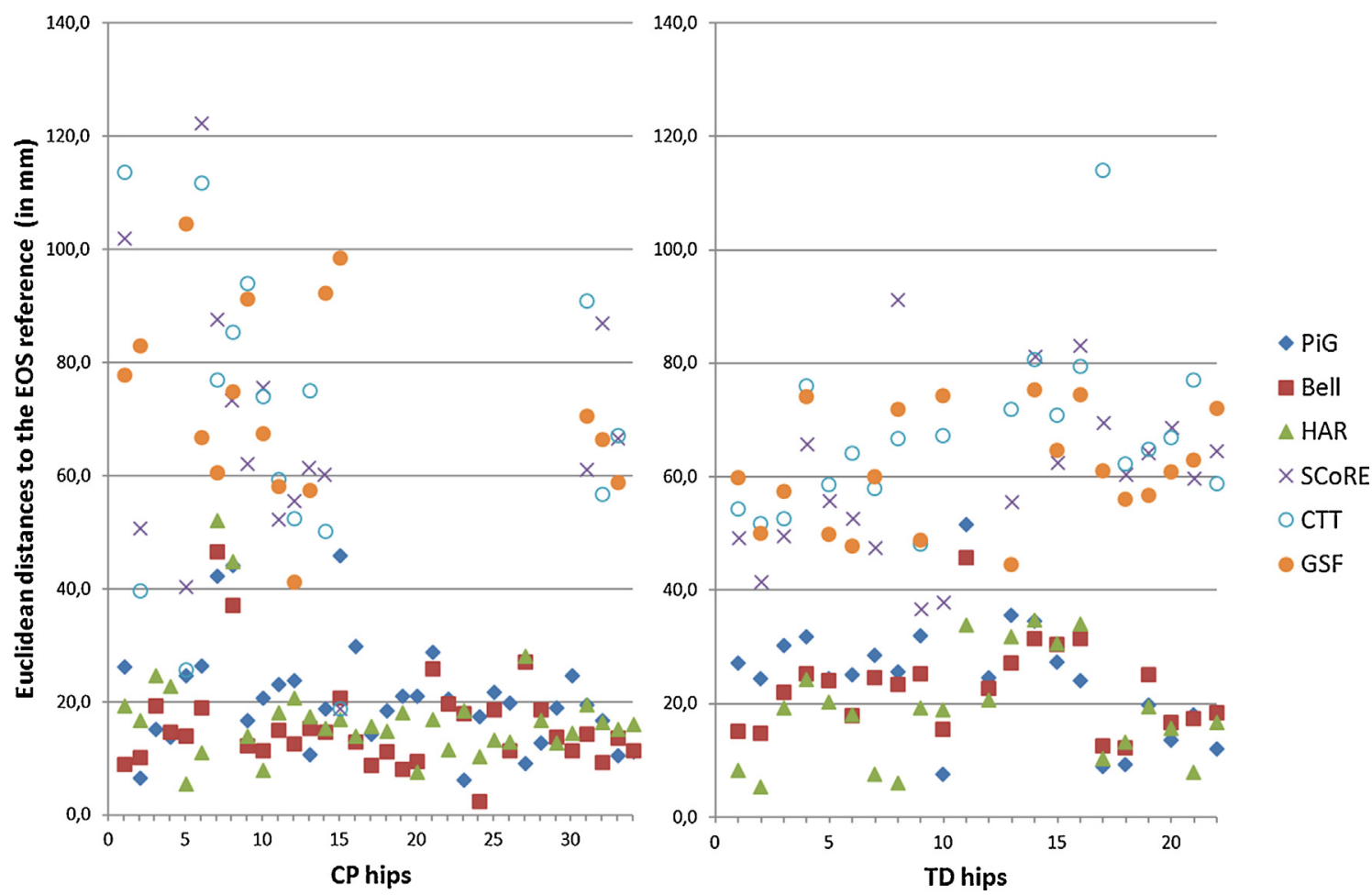

Fig. 2. Euclidean distances to the EOS reference of the 6 hip joint center localization techniques in 34 hips of children with cerebral palsy and 22 hips of typically developing children.
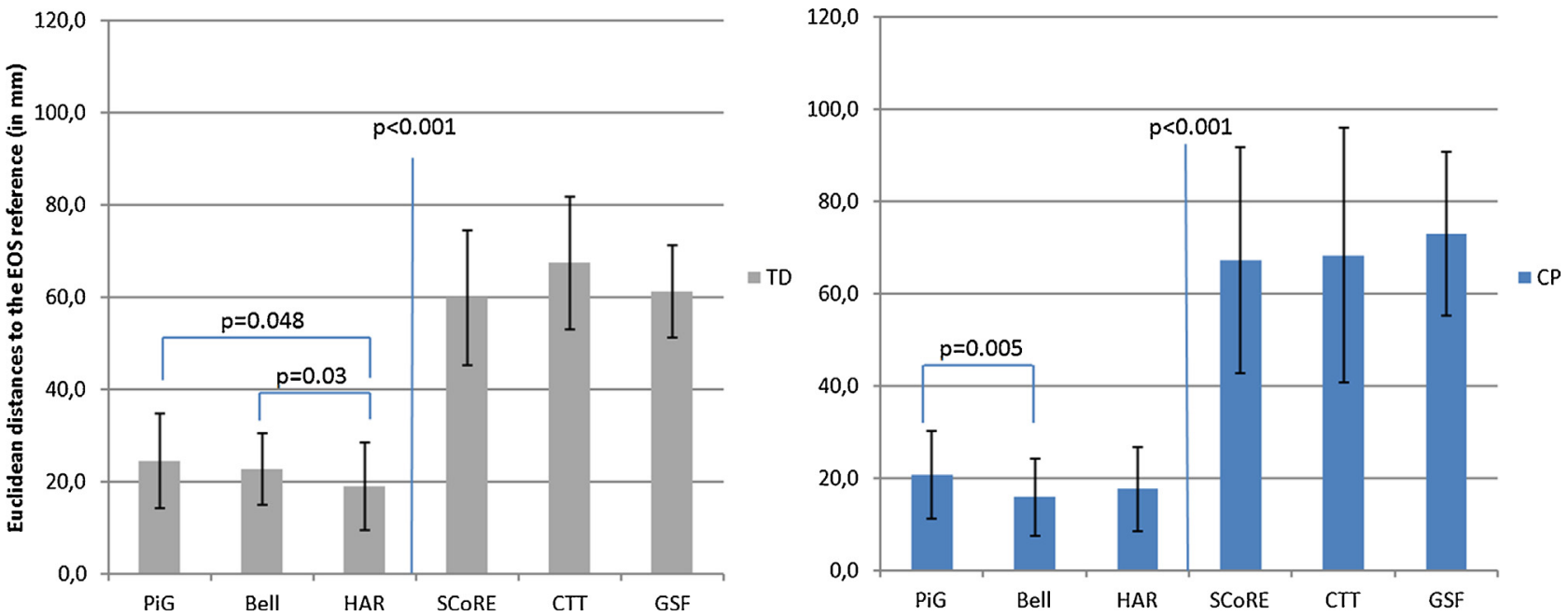

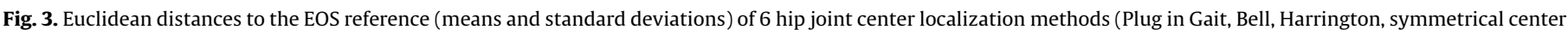
of rotation estimate, center transformation technique, geometrical sphere fitting) in typically developing children (left) and children with cerebral palsy (right).

to be the most accurate in TD children. Both the Harrington and Bell methods were equally accurate in the CP group.

Previous validation studies of $\mathrm{HJC}$ localization techniques have been based on either fluoroscopy or 3D ultrasound (3DUS) $[9,15,17,25,26]$. While the former method is known to expose the subjects to a high dose of radiation, the latter does not. The 3D EOS $^{\circledR}$ imaging technique does entail a certain dose of radiation to the subject; however, this dose is known to be 2-5 times less than the conventional X-ray [27]. Moreover, it has been shown that this technique is more reliable than $3 \mathrm{D}$ ultrasound $(2.9 \pm 1.3 \mathrm{~mm}$ vs. $4 \pm 2 \mathrm{~mm}$ ) [16,19] and crucially, allows the simultaneous acquisition of: the 3D subject-specific reconstruction of the skeletal segments, the external reflective markers and the HJC reference, which contributes to the reduction of errors.
Our results were comparable to those obtained by Peters et al. [26], where predictive and functional HJC localization methods were compared to a 3D ultrasound-based image technique in $\mathrm{CP}$ children. The authors found that the distance between the reference and the HAR technique was approximately $14 \pm 8 \mathrm{~mm}$. While both Harrington and Plug in Gait techniques showed similar results between our study and Peters et al.'s study, the large difference of errors on functional methods (i.e. GSF technique, current study $\approx 66 \mathrm{~mm}$ vs. Peters study $\approx 20 \mathrm{~mm}$ ) could be due to the difference in the image based technique used and to the fact that the star arc acquisition was assisted by an operator in Peters et al.'s study.

In a recent study, Sangeux et al. [20] used the $\operatorname{EOS}^{\circledR}$ system to validate the hip joint center localization techniques in healthy adults. The Harrington method showed the best result when a 


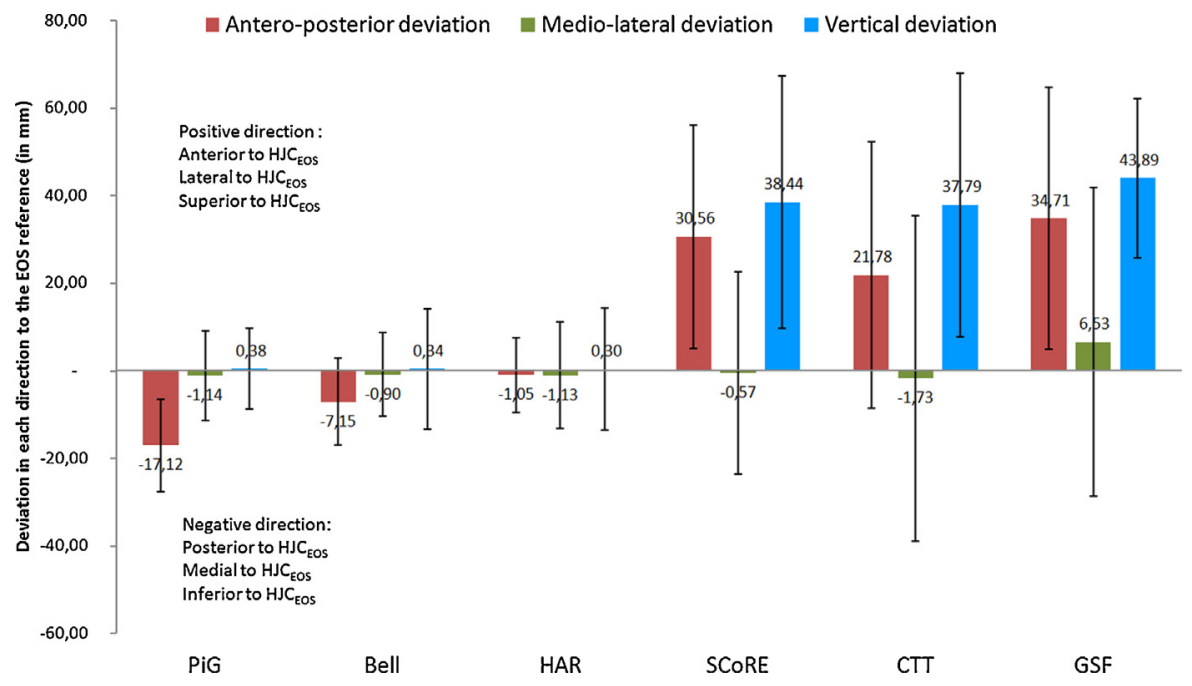

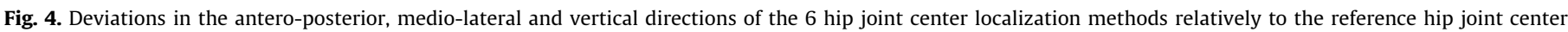
obtained from EOS (means and standard deviations).

reduced range of motion $\left(<30^{\circ}\right)$ was performed during hip calibration with Euclidean distances to EOS $^{\circledR}$ reference similar to those obtained in our study $(17 \mathrm{~mm})$. However, the authors showed that with a large performed ROM $\left(>30^{\circ}\right)$ during hip star arc movement, the geometrical sphere fitting method located the HJC closer to the $\operatorname{EOS}^{\circledR}$ reference $(11 \mathrm{~mm})$ compared to the other methods. The differences in the results obtained between adults (Sangeux et al.) and children (current study) using the same image based reference technique could be related to the smaller segment of the thigh in children; the smaller segment moves the thigh markers closer to the hip, which might increase the noise when sphere fitting or transformational techniques are used to locate the center of rotation. Moreover, even though the $\mathrm{CP}$ group performed a significantly lower range of motion than the TD group in this study, average range of motion in both groups was higher than $30^{\circ}$. Furthermore, the ANCOVA test showed that the ROM was not a confounding factor on the errors on the HJCs calculated by the functional methods.

The propagation of the errors in the localization of the hip joint center on kinematics and kinetics has been previously evaluated in the literature $[24,28]$. It was shown that hip joint center misplacement could result in significant errors on both hip and knee kinematics and kinetics. A threshold of $30 \mathrm{~mm}$ was defined as the limit of acceptable errors on the hip joint center localization, below which the kinematics and kinetics are not significantly affected $[20,24]$. In the current study, the proportion of errors on hip localization that fall above the threshold of $30 \mathrm{~mm}$ was shown to be high in functional methods. Therefore, the errors on HJC localization techniques could lead to erroneous gait data and thus to an inadequate clinical interpretation. While different studies have shown that the functional methods are the best for the localization of the HJC $[9,20,25,26]$, the current study has thus shown that the functional methods are less accurate than the predictive ones in both CP and TD children.

In this study, deviations to the reference of $\mathrm{HJC}$ localization techniques were mostly comparable to those obtained in the literature in the case of adult subjects, using the EOS imaging technique [20], except for a few differences. While the Plug in Gait (PiG) technique was shown to place the HJC anterior to the reference in the previous study on adults $(\approx+10 \mathrm{~mm})$, we found that the PiG technique was posterior to the reference in the case of children $(\approx-14 \mathrm{~mm})$. This could be due to the fact that the regression equation used by the Davis method, which was computed from adult cadavers [7], is inadequate for use in children. Moreover, the results obtained in our study, in the case of children, showed that the functional techniques placed the hip joint center further anteriorly to the reference compared to adults $(\approx 30 \mathrm{~mm}$ in children vs. $12-25 \mathrm{~mm}$ in adults [20]) and more superiorly.

As previously indicated, the significant deviations of hip joint center localization techniques from the gold standard used in this study (EOS ${ }^{\circledR}$ system) would implicate major errors on kinematics and kinetics [28]. These errors can influence various uses of gait analysis such as evidence-based decision-making, choice of dosages of botulinum toxin injections and/or musculoskeletal surgery evaluation.

In conclusion, a novel technique was used to validate predictive and functional methods of hip joint center localization used in the setting of gait analysis in children with cerebral palsy and typically developing children. The 3D EOS ${ }^{\mathbb{R}}$ imaging technique has shown that the predictive methods perform better than the functional methods and that the Harrington regression method has the best results in both $\mathrm{CP}$ and TD children. While the Harrington method showed slightly better results than the Bell method, both could be used with quasi-equivalent performance. Moreover, the bias was quantified for each of them in each direction, which can allow correction of those predictive methods. Since the Harrington method was based on data from adults and children, the computation of a children-specific regression equation could better predict the location of the hip joint center to be used in gait analysis. When available, the $\operatorname{EOS}^{\mathbb{R}}$ system could be an alternative to estimation methods by detecting the exact location of the hip joint center, which can be integrated in the computation of the kinematic waveforms.

\section{Acknowledgments}

This study was funded by the Research Council of the University of Saint-Joseph (grant \# FM244) and the CEDRE Lebanese-French Governmental Cooperation for Research (grant \# 11SCIF44/L36).

\section{References}

[1] Davids JR. Quantitative gait analysis in the treatment of children with cerebral palsy. J. Pediatr. Orthop. 2006;26:557-9. http://dx.doi.org/10.1097/01.bpo. 0000226284.46943.a3.

[2] Narayanan UG. The role of gait analysis in the orthopaedic management of ambulatory cerebral palsy. Curr. Opin. Pediatr. 2007;19:38-43. http:// dx.doi.org/10.1097/MOP.0b013e3280118a6d. 
[3] Cappozzo A, Della Croce U, Leardini A, Chiari L. Human movement analysis using stereophotogrammetry: Part 1. Theoretical background. Gait Posture 2005;21:186-96. http://dx.doi.org/10.1016/j.gaitpost.2004.01.010.

[4] Wu G, Cavanagh PR. ISB recommendations in the reporting for standardization of kinematic data. J. Biomech. 1995;28:1257-61. http://dx.doi.org/10.1016 0021-9290(95)00017-C.

[5] Wu G, Siegler S, Allard P, Kirtley C, Leardini A, Rosenbaum D, et al. ISB recommendation on definitions of joint coordinate system of various joints for the reporting of human joint motion - Part I. Ankle, hip, and spine. J. Biomech. 2002;35:543-8. http://dx.doi.org/10.1016/S0021-9290(01)00222-6.

[6] Bell AL, Brand RA, Pedersen DR. Prediction of hip joint centre location from external landmarks. Hum. Mov. Sci. 1989;8:3-16. http://dx.doi.org/10.1016 0167-9457(89)90020-1.

[7] Davis RB, Ounpuu S, Tyburski D, Gage JR. A gait analysis data collection and reduction technique. Hum. Mov. Sci. 1991;10:575-87. http://dx.doi.org 10.1016/0167-9457(91)90046-Z.

[8] Harrington ME, Zavatsky AB, Lawson SEM, Yuan Z, Theologis TN. Prediction of the hip joint centre in adults, children, and patients with cerebral palsy based on magnetic resonance imaging. J. Biomech. 2007;40:595-602. http:/ dx.doi.org/10.1016/j.jbiomech.2006.02.003.

[9] Leardini A, Cappozzo A, Catani F, Toksvig-Larsen S, Petitto A, Sforza V, et al Validation of a functional method for the estimation of hip joint centre location. Biomech. 1999;32:99-103. http://dx.doi.org/10.1016/S0021-9290(98)00148-1.

[10] Schwartz MH, Rozumalski A. A new method for estimating joint parameters from motion data. J. Biomech. 2005;38:107-16. http://dx.doi.org/10.1016 j.jbiomech.2004.03.009.

[11] Ehrig RM, Taylor WR, Duda GN, Heller MO. A survey of formal methods for determining the centre of rotation of ball joints. J. Biomech. 2006;39:2798809. http://dx.doi.org/10.1016/j.jbiomech.2005.10.002.

[12] Piazza SJ, Erdemir A, Okita N, Cavanagh PR. Assessment of the functional method of hip joint center location subject to reduced range of hip motion. J. Biomech. 2004;37:349-56. http://dx.doi.org/10.1016/S0021-9290(03)00288-4.

[13] Chang LY, Pollard NS. Constrained least-squares optimization for robus estimation of center of rotation. J. Biomech. 2007;40:1392-400. http:// dx.doi.org/10.1016/j.jbiomech.2006.05.010.

[14] Cereatti A, Donati M, Camomilla V, Margheritini F, Cappozzo A. Hip joint centre location: an ex vivo study. J. Biomech. 2009;42:818-23. http://dx.doi.org 10.1016/j.jbiomech.2009.01.031.

[15] Bell AL, Pedersen DR, Brand RA. A comparison of the accuracy of several hip center location prediction methods. J. Biomech. 1990;23:617-21. http: dx.doi.org/10.1016/0021-9290(90)90054-7.

[16] Peters A, Baker R, Sangeux M. Validation of 3-D freehand ultrasound for the determination of the hip joint centre. Gait Posture 2010;31:530-2. http: dx.doi.org/10.1016/j.gaitpost.2010.01.014
[17] Sangeux M, Peters A, Baker R. Hip joint centre localization: evaluation on normal subjects in the context of gait analysis. Gait Posture 2011;34:324-8. http://dx.doi.org/10.1016/j.gaitpost.2011.05.019.

[18] Dubousset J, Charpak G, Skalli W, Deguise J, Kalifa G. EOS: a new imaging system with low dose radiation in standing position for spine and bone and joint disorders. J. Musculoskelet. Res. 2010;13:1-12. http://dx.doi.org/10. $1142 /$ S0218957710002430.

[19] Pillet H, Sangeux M, Hausselle J, El Rachkidi R, Skalli W. A reference method for the evaluation of femoral head joint center location technique based on external markers. Gait Posture 2014;39:655-8. http://dx.doi.org/10.1016/ j.gaitpost.2013.08.020

[20] Sangeux M, Pillet H, Skalli W. Which method of hip joint centre localisation should be used in gait analysis? Gait Posture 2014;40:20-5. http://dx.doi.org/ 10.1016/j.gaitpost.2014.01.024

[21] Camomilla V, Cereatti A, Vannozzi G, Cappozzo A. An optimized protocol for hip joint centre determination using the functional method. J. Biomech. 2006;39:1096-106. http://dx.doi.org/10.1016/j.jbiomech.2005.02.008.

[22] Chaibi Y, Cresson T, Aubert B, Hausselle J, Neyret P, Hauger O, et al. Fast 3D reconstruction of the lower limb using a parametric model and statistical inferences and clinical measurements calculation from biplanar X-rays. Comput. Methods Biomech. Biomed. Eng. 2012;15:457-66. http://dx.doi.org/10. 1080/10255842.2010.540758.

[23] Assi A, Chaibi Y, Presedo A, Dubousset J, Ghanem I, Skalli W. Three-dimensional reconstructions for asymptomatic and cerebral palsy children's lower limbs using a biplanar X-ray system: a feasibility study. Eur. J. Radiol. 2013;82:2359-64. http://dx.doi.org/10.1016/j.ejrad.2013.07.006.

[24] Stagni R, Leardini A, Cappozzo A, Grazia Benedetti M, Cappello A. Effects of hip joint centre mislocation on gait analysis results. J. Biomech. 2000;33:1479-87. http://dx.doi.org/10.1016/S0021-9290(00)00093-2.

[25] Hicks JL, Richards JG. Clinical applicability of using spherical fitting to find hip joint centers. Gait Posture 2005;22:138-45. http://dx.doi.org/10.1016/j.gaitpost.2004.08.004.

[26] Peters A, Baker R, Morris ME, Sangeux M. A comparison of hip joint centre localisation techniques with 3-DUS for clinical gait analysis in children with cerebral palsy. Gait Posture 2012;36:282-6. http://dx.doi.org/10.1016/j.gaitpost.2012.03.011.

[27] Dietrich TJ, Pfirrmann CWA, Schwab A, Pankalla K, Buck FM. Comparison of radiation dose, workflow, patient comfort and financial break-even of standard digital radiography and a novel biplanar low-dose X-ray system for upright full-length lower limb and whole spine radiography. Skeletal Radiol. 2013;42:959-67. http://dx.doi.org/10.1007/s00256-013-1600-0.

[28] Kiernan D, Malone A, O'Brien T, Simms CK. The clinical impact of hip joint centre regression equation error on kinematics and kinetics during paediatric gait. Gait Posture 2015;41:175-9. http://dx.doi.org/10.1016/j.gaitpost.2014.09.026. 